\title{
CONSUMO DE SAL DE ADIÇÃO EM ALUNOS DO ENSINO MÉDIO ANTES E APÓS CONSCIENTIZAÇÃO SOBRE O CONSUMO DE SÓDIO
}

Flávia Queiroga Aranha ${ }^{1}$ Aline de Cássia Albano ${ }^{2}$

Aline de Oliveira Martins

Caroline de Barros Gomes

\section{Resumo}

Hipertensão arterial sistêmica (HAS) é uma condição clínica multifatorial caracterizada por níveis elevados e sustentados de pressão arterial (PA), sendo a presença de PA elevada na infância como fator preditor de HAS na vida adulta. Estudos têm mostrado correlação positiva entre o sal ingerido na dieta e valores de PA. Este estudo objetiva avaliar o consumo de sal de adição antes e após conscientização com alunos do ensino médio. Esta avaliação ocorreu durante três dias no refeitório, onde os alunos receberam folder ilustrativo com informações sobre o consumo de sódio; exposição de cartaz ilustrando alimentos com sua respectiva quantidade de sódio e equivalência em cloreto de sódio; display exemplificando a quantidade máxima recomendada de consumo por dia. Durante cinco dias, antes e após a conscientização, foram contabilizados os sachês de sal. As análises estatísticas foram realizadas por teste $T$ Student, com intervalo de confiança de $95 \%$. Durante o período foram servidas, em média, 128 refeições. Antes da conscientização foram consumidos, em média, $820 \mathrm{mg}$ de sal e $330 \mathrm{mg}$ de sódio, por indivíduo; e após, 640mg e 260mg, respectivamente (p=0,043). Estratégias de Educação Nutricional simples e mesmo que em curto prazo foram efetivas para a melhoria do consumo de sal de adição em adolescentes.

Palavras-chave: Sódio; Educação Nutricional; Adolescente.

\section{Introdução}

A hipertensão arterial sistêmica (HAS) é uma condição clínica multifatorial caracterizada por níveis elevados e sustentados de pressão arterial (PA). Inquéritos populacionais em cidades brasileiras apontaram uma prevalência de HAS acima de 30\% (SBC, 2010).

Embora predomine na idade adulta, a hipertensão arterial em crianças e adolescentes não é desprezível. Ela varia amplamente nos relatos de diversos autores nacionais e estrangeiros, de $1 \%$ a $13 \%$, dependendo, sobretudo, da metodologia empregada (SALGADO e CARVALHAES, 2003).

Em crianças e adolescentes, a hipertensão arterial parece desenvolver-se mais rapidamente, sendo a presença de PA elevada na infância fator preditor de hipertensão arterial na vida adulta. A manifestação da PA elevada depende também de fatores ambientais como

\footnotetext{
${ }^{1}$ Professora Doutora do Departamento de Educação do Instituto de Biociências de Botucatu - UNESP.

${ }^{2}$ Graduandas do Curso de Nutrição do Instituto de Biociências de Botucatu - UNESP. 
dieta, atividade física, estresse, envelhecimento, história familiar, raça, gênero, peso e tabagismo (RINALDI et al., 2012; MOLINA et al., 2003).

Não são conhecidos completamente os mecanismos responsáveis pela elevação da pressão arterial em crianças, entretanto o excesso de peso e alto consumo de sódio são fatores de risco para esta condição. Com a crescente prevalência de excesso de peso em toda a população, inclusive entre crianças e adolescentes, tem acrescido o número de casos de hipertensão arterial primária nesta faixa etária. Além disso, estudos clínicos e experimentais tem mostrado correlação positiva entre o sal ingerido na dieta e valores de PA, reafirmando que o consumo elevado de cloreto de sódio na dieta está associado a valores de PA mais altos (HOFFMANN et al., 2010; RUIVO e HEIMANN, 2003; RINALDI et al., 2012).

A principal fonte de sódio na alimentação é o sal comum ( $40 \%$ de sódio), que é empregado rotineiramente na cozinha, no processamento dos alimentos e à mesa. Segundo a Pesquisa de Orçamentos Familiares - POF 2008-2009, mais de 70\% dos adolescentes brasileiros tiveram ingestão de sódio superior ao valor máximo de ingestão tolerável (NAKASATO, 2004; BRASIL, 2010).

Diante da relevância do problema, medidas de contenção do avanço da hipertensão se justificam plenamente, não apenas com ênfase no diagnostico e tratamento dos indivíduos já acometidos e/ou na identificação daqueles mais suscetíveis ao seu desenvolvimento, mas, principalmente, na implementação de estratégias no sentido de prevenir o aparecimento da doença (WILLIAMS, 2010; MAGALHÃES, 2010).

A escola é o local ideal para a prática de ações de promoção a alimentação saudável já que a maioria das crianças passa grande parte do tempo neste ambiente. Somado a este fato, as escolas munem os estudantes de ferramentas necessárias para a compreensão das orientações de saúde disseminadas pelos variados meios de comunicação (MARTINEZ, 1996).

Deste modo, o presente estudo objetiva avaliar o consumo de sal de adição entre alunos do ensino médio, antes e após a conscientização sobre o consumo de sódio.

\section{Material e método}

Trata-se de um estudo transversal em que foi avaliado o consumo de sal de adição sachês, antes e após conscientização sobre o consumo de sódio. O público alvo foram estudantes do primeiro grau do Ensino Médio de colégio mantido por Instituto Social de uma empresa de grande porte, em município do interior de São Paulo. A alimentação destes alunos 
é fornecida por uma empresa terceirizada, no serviço de self-service parcial, com porcionamento do prato principal e da sobremesa. O cardápio é composto por três tipos de saladas, um prato principal, uma guarnição, arroz, feijão, sopa, sobremesa (doce ou fruta) e suco. Os temperos adicionais podem ser consumidos à vontade: azeite, vinagre, shoyo, molho de pimenta e sachês de $0,8 \mathrm{~g}$ de Cloreto de Sódio.

A conscientização sobre o consumo de sódio ocorreu durante três dias no período que os alunos se mantinham no refeitório para o almoço. O slogan da conscientização foi: "Sódio: você está consumindo a quantidade recomendada?". No primeiro dia, ao saírem do refeitório, todos os alunos receberam folder ilustrativo, elaborado especialmente para este estudo, com informações acerca do Sódio, sua importância e consequências do consumo excessivo. O folder ainda continha tabela com alimentos e sua respectiva quantidade em sódio e equivalência em Cloreto de Sódio, cuja quantidade foi apresentada em cartaz no segundo dia da conscientização, fixado no início da fila de distribuição das refeições. Além do cartaz houve explicação e esclarecimentos de dúvidas a grupos de alunos participantes durante a espera da distribuição das refeições. No mesmo dia, ao lado do molho shoyo, foi locado display ilustrando a quantidade equivalente em Cloreto de Sódio em duas colheres de sopa deste tempero. Foi observado amplo consumo deste pelos alunos e, além disto, o molho shoyo é um alimento rico em sódio. Finalmente, no terceiro dia, o cartaz foi mantido fixo ao início dos balcões de distribuição e, ao lado dos sachês de sal, foi locado display ilustrativo com a quantidade máxima de consumo de sachês de sal por dia, com o lembrete de que a maioria das preparações e alimentos também possui sal em sua composição.

Durante cinco dias, antes e após a conscientização sobre o consumo de sódio, foi contabilizado o consumo de sachês de sal pela diferença entre a quantidade de sachês no início e no final do almoço somada a quantidade de sachês devolvida fechada na devolução das bandejas. Não foi contabilizado o sal que porventura estivesse restante nos sachês nas bandejas. Em todos os dias (antes e depois) foi contabilizada a quantidade de alunos que almoçaram no refeitório, para a realização do cálculo de consumo de sal de adição per capita. Posteriormente, considerando a composição de $40 \%$ de sódio no Cloreto de Sódio, foi estimada a ingestão de sódio per capita.

As análises estatísticas foram realizadas no programa SPSS v.19 2012, sendo a diferença entre o consumo, antes e depois, estimada por teste T-Student para amostras pareadas, com intervalo de confiança de $95 \%$. 


\section{Resultados}

Durante o período estudado (antes e após conscientização) foram servidos em média 128 comensais/refeições. A quantidade dos sachês de sal consumidos pelos estudantes antes da conscientização sobre o consumo de sódio é apresentada na tabela 1 . O valor médio de sal e sódio de adição consumidos nas refeições antes da conscientização foi de 820 e 330 miligramas, respectivamente.

Tabela 1. Consumo de sal e sódio de adição antes da conscientização sobre o consumo de sódio.

\begin{tabular}{ccccc}
\hline Dia & $\begin{array}{c}\text { Consumo em } \\
\text { sachês }\end{array}$ & Comensais & $\begin{array}{c}\text { Gramas de sal de } \\
\text { adição por indivíduo }\end{array}$ & $\begin{array}{c}\text { Gramas de Sódio de } \\
\text { adição por indivíduo }\end{array}$ \\
\hline 1 & 134 & 131 & 0,82 & 0,33 \\
2 & 175 & 133 & 1,05 & 0,42 \\
3 & 115 & 131 & 0,70 & 0,28 \\
4 & 135 & 129 & 0,84 & 0,33 \\
5 & 109 & 128 & 0,68 & 0,27 \\
\hline MÉDIA & 133,6 & 130,4 & 0,82 & 0,33 \\
\hline
\end{tabular}

Após as atividades de conscientização (tabela 2), o valor médio de sal e sódio de adição consumidos nas refeições foi de 640 e 260 miligramas, respectivamente. Segundo teste $T$ Student para amostras pareadas houve redução estatisticamente significante no consumo de sódio de adição pelos estudantes, como apresentado na figura 1.

Tabela 2. Consumo de sal e sódio de adição após conscientização sobre o consumo de sódio.

\begin{tabular}{ccccc}
\hline Dia & $\begin{array}{c}\text { Consumo em } \\
\text { sachês }\end{array}$ & Comensais & $\begin{array}{c}\text { Gramas de sal de } \\
\text { adição por indivíduo }\end{array}$ & $\begin{array}{c}\text { Gramas de Sódio de } \\
\text { adição por indivíduo }\end{array}$ \\
\hline 1 & 104 & 129 & 0,64 & 0,26 \\
2 & 103 & 130 & 0,63 & 0,25 \\
3 & 93 & 125 & 0,60 & 0,24 \\
4 & 119 & 130 & 0,73 & 0,29 \\
5 & 86 & 117 & 0,59 & 0,24 \\
\hline MÉDIA & 101 & 126,2 & 0,64 & 0,26 \\
\hline
\end{tabular}

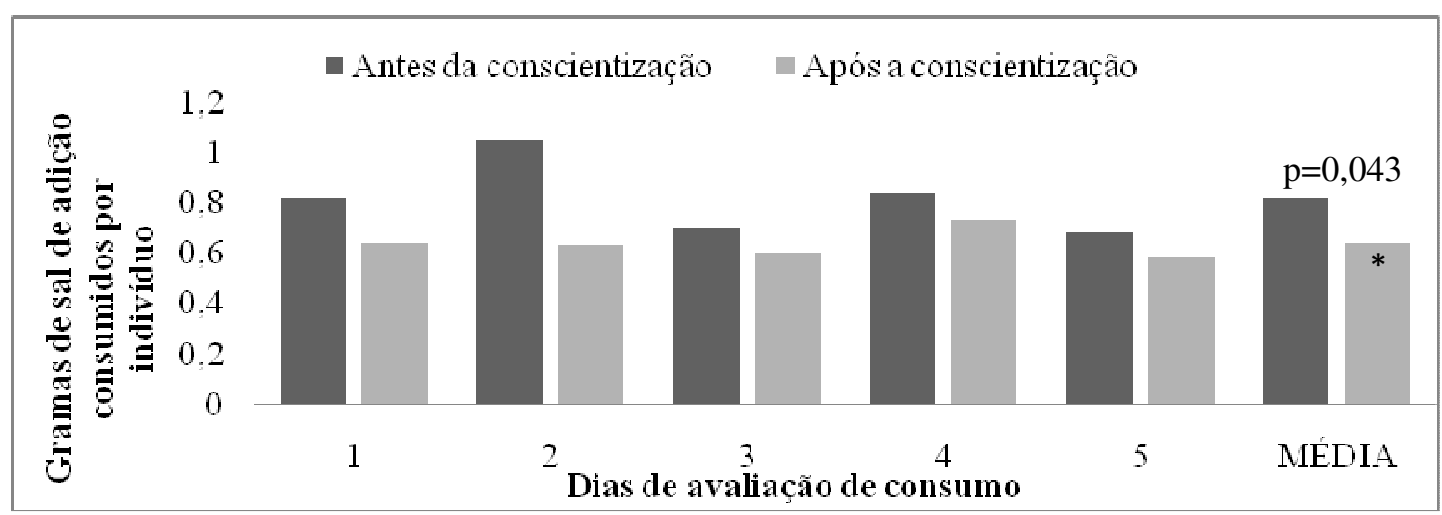

Figura 1. Consumo em gramas de sal de adição no período de avaliação, antes e após conscientização sobre consumo de sódio. 


\section{Discussão}

Artigo recentemente publicado encontrou inadequação no consumo de vitaminas e minerais entre adolescentes, população deste estudo, com ênfase para o consumo de sódio muito além da recomendação de $2000 \mathrm{mg}$ por dia, sendo a pior situação entre meninos de 14 a 18 anos, com mediana de consumo de $3.491 \mathrm{mg} / \mathrm{dia}$ (VEIGA et al., 2013). PIOLTINE et al. (2013), em estudo realizado com 48 adolescentes de uma escola privada de São Paulo, também constatou elevada ingestão de sódio por 37,5\% dos alunos de ambos os gêneros.

Do total de sódio ingerido durante o dia, o valor de sal de adição deveria corresponder a 15\%, ou seja, 300 mg/dia (AUGUSTO et al., 1999 apud SPINELLI e KOGA,2007). Antes da conscientização sobre o consumo de sódio, os alunos estudados consumiam $110 \%$ desta recomendação de sal de adição e, após este período, consumiram 86,7\% desta recomendação, redução estatisticamente significativa, contudo se pode considerar ainda elevada, já que este valor foi consumido apenas no almoço. A importância desta diminuição na ingestão é reforçada por estudos que apresentam que reduções, mesmo modestas, no consumo de sódio acarretam efeitos benéficos sobre a saúde das pessoas, e determinariam importante arrefecimento nos gastos com o tratamento de doenças (HE e MACGREGOR, 2004; WANG e LABARTHE, 2011). HE e MACGREGOR (2004) encontraram em sua metanálise que durante quatro ou mais semanas a diminuição da ingestão de sal já tem efeito significativo na diminuição da pressão arterial em indivíduos normo ou hipertensos.

Em avaliação do consumo de sal em uma Unidade de Alimentação e Nutrição de Suzano - SP, com a mesma metodologia de contagem de sachês de sal, SPINELLI e KOGA (2007) encontraram consumo médio 0,59 gramas de sal de adição por pessoa no almoço, valor inferior ao encontrado no presente estudo tanto antes como após a conscientização. A literatura é escassa quanto à avaliação do consumo de sódio de adição, especialmente entre e a população adolescente, como é o caso neste estudo.

Consideramos que a contagem dos sachês de sal consumidos, sem a contabilização do que porventura sobrou nas embalagens e foi desprezado, é a limitação deste estudo, pois se pode estar superestimando o consumo.

Apesar de o presente estudo ter objetivado apenas a conscientização sobre o consumo de sódio, em um período de tempo bastante curto foi capaz de alcançar mudanças positivas e negativas nos hábitos alimentares de adolescentes, de acordo com os parâmetros deste estudo. 
Mudanças positivas nos hábitos alimentares de adolescentes também foram obtidas em estudo desenvolvido em Pernambuco, no qual adolescentes obesos que receberam intervenção nutricional, psicológica, clínica e física durante doze semanas e apresentaram redução significativa no consumo de energia, lipídios, proteínas e sódio (ANDRADE et al., 2012).

Além do sódio de adição, sabidamente, o atual grande consumo de produtos industrializados ricos em sódio é importante fator que contribui para o elevado consumo de sódio na população brasileira. O público adolescente ganha destaque neste consumo já que são atraídos pela praticidade, propagandas e "modernidade" que estes produtos apresentam. Estudo realizado com adolescentes de colégio do município de Petrópolis-RJ conclui que, embora os alunos pesquisados conhecessem sobre os hábitos alimentares saudáveis, a maioria revelava não os seguir e possuía uma cultura de alimentação com preparações rápidas (SILVA, 2012).

Neste sentido, o ambiente escolar, por ser local de ensino para formação de comportamentos e conhecimentos, é um importante espaço para a educação nutricional (BRUG, 2008). A Educação Nutricional, como método de ensino, é uma ferramenta simples e de baixo custo na promoção à saúde, sendo que estas ações devem sempre ser apresentadas como agentes de mudança pelo nutricionista, independente da sua área de atuação (TURANO \& ALMEIDA, 1999).

\section{Conclusão}

Estratégias de Educação Nutricional simples, mesmo que realizadas em curto período de tempo, foram efetivas para a melhoria do consumo de sal de adição durante o almoço em indivíduos adolescentes. Destaca-se a importância da realização permanente de projetos de educação nutricional nas escolas, sejam públicas ou privadas, sendo o nutricionista, independente de sua área de atuação, responsável como educador, atuando como promotor da saúde e de hábitos alimentares saudáveis.

\section{Referências}

ANDRADE, M. L. S. S.; SILVA, H. J. G.; LOFRANO-PRADO, M. C.; FERREIRA, M. N. L.; LINS, T. A.; BURKHARDT, R. C.; PRADO, W. L. Efeitos da intervenção multidisciplinar sobre a ingestão alimentar em adolescentes obesos. ConScientiae Saúde, v. 11, n. 2, p.289-295, 2012.

BRASIL. Instituto Brasileiro de Geografia e Estatística. Pesquisa de Orçamentos Familiares 2008-2009. Rio de Janeiro, 2010. 
BRUG, J. Determinants of healthy eating: motivation, abilities and environmental opportunities. Family Pract. Adv. v.25, p.i50-i55, 2008.

HE, F.J.; MACGREGOR, G.A. Effect of longer-term modest salt reduction on blood pressure. Cochrane DatabaseSyst Rev., v.3, CD004937, 2004.

HOFFMANN, M.; SILVA, A. C. P.; SIVIERO, J. Prevalência de hipertensão arterial sistêmica e inter-relações com sobrepeso, obesidade, consumo alimentar e atividade física, em estudantes de escolas municipais de Caxias do Sul. Pediatria (São Paulo), v. 32, n. 3, p. 16372, 2010.

MAGALHÃES, M. E. C.; BRANDÃO, A. A.; POZZAN, R.; CAMPANA, E. M. G.; FONSECA, F. L.; PIZZI, O. L.; BRANDÃO, A. P. Prevenção da hipertensão arterial: para quem e quando começar? Rev. Bras. Hipertens., v.17, n. 2, p. 93-97, 2010.

MARTÍNEZ, A. M. La escuela: unespacio de promocion de salud. PsicolEsc Educ. v.1, p.1924, 1996.

MOLINA, M. C. B.; CUNHA, R. S.; HERKENHOFF, L. F.; MILL. J. G. Hipertensão arterial e consumo de sal em população urbana. Rev. Saúde Pública, São Paulo, v.37, n.6, dez. 2003.

NAKASATO, M. Sal e hipertensão arterial. Rev. Bras. Hipertens., v. 11, n. 2, p. 95-97. 2004.

PIOLTINE, M. B.; PATERNEZ, A. C. A. C.; MORIMOTO, J. M. Perfil dietético e risco cardiovascular em adolescentes de uma escola privada de São Paulo. Rev. Ciência \& Saúde, Porto Alegre, v. 6, n. 3, p. 149-156, set./dez. 2013

RINALDI, A. E. M. et al. Prevalência de pressão arterial elevada em crianças e adolescentes do ensino fundamental. Rev. Paul. Pediatr, v. 30, n.1, p. 79-86. 2012.

RUIVO, G. F.; HEIMANN, J. C. Revisão: O efeito do sal na resistência à insulina evidências clínicas e experimentais. J. Bras. Nefrol., v. 25, n. 1, p. 34-40. 2003.

SAlGADO, C. M.; CARVALHAES, J. T. A. Hipertensão arterial na infância. J. Pediatr., v. 79, supl. 1, 2003.

SILVA, J.G.; TEIXEIRA, M.L.O.; FERREIRA, M.A. Alimentação e saúde: sentidos atribuídos por adolescentes. Esc. Anna Nery (impr.), v.16, n.1, p.88-95, 2012.

SOCIEDADE BRASILEIRA DE CARDIOLOGIA / SOCIEDADE BRASILEIRA DE HIPERTENSÃO / SOCIEDADE BRASILEIRA DE NEFROLOGIA. VI Diretrizes Brasileiras de Hipertensão. Arq. Bras. Cardiol., Rio de Janeiro, v. 95, nº 1, p. 1-51, 2010.

SPINELLI, M. G. N.; KOGA, T. T. Salt intake evaluation in a food service. Nutrire: rev. Soc. Bras. Alim.Nutr.= J. Brazilian Soc. Food Nutr., v. 32, n. 2, p. 15-27, ago. 2007.

TURANO, W.; ALMEIDA, C. C. C. A. Educação Nutricional. In: GOUVEIA, E. L. C. Nutrição, Saúde e Comunidade. 2. ed. Rio de Janeiro: Revinter, p.57-77,1999. 
VEIGA, G. V.; DA COSTA, R. S.; ARAÚJO, M. C.; SOUZA, A. M.; BEZERRA, I. N.; BARBOSA, F. S.; SICHIERI, R.; PEREIRA, R. A. Inadequação do consumo de nutrientes entre adolescentes brasileiros. Rev. Saúde Pública, v.47, supl.1, p.212s-221s, 2013.

WANG, G.; LABARTHE, D. The cost-effectiveness of interventions designed to reduce sodium intake. J. Hypertens., v.29, n. 9, p. 1693-1699, 2011.

WILLIAMS, B. The year in hypertension. J Am Coll Cardiol, v. 55, n. 1, p. 66-73, 2010.

\title{
CONSUMPTION OF SALT OF ADDITION IN HIGH SCHOOL STUDENTS BEFORE AND AFTER OF AWARENESS SODIUM INTAKE
}

\begin{abstract}
High blood pressure (HBP) is a multifactorial clinical condition characterized by high and sustained levels of blood pressure (BP), and the presence of elevated BP in childhood is predictor of hypertension in adult life. Studies have shown a positive correlation between sodium chloride ingested in the diet and blood pressure values. This study aims to evaluate the consumption of added salt before and after awareness of risk, by high school students. This occurred for three days in the refectory, where students received illustrative brochure with information about sodium intake; exhibition of a poster illustrating foods with their respective equivalent amount of sodium and sodium chloride, exemplifying the maximum recommended amount of consumption per day. For five days, before and after gaining awareness, it the amount of consumption of sachets of salt was accounted. Statistical analyzes were performed by Student's Test, with a confidence interval of 95\%. During the study period were served on average 128.3 meals. Before the awareness of sodium consumption of risks, of $820 \mathrm{mg}$ and $330 \mathrm{mg}$ sodium salt was consumed an average per individual, and thereafter, 640mg and 260mg, respectively $(\mathrm{p}=0,043)$. Simple Nutrition Education Strategies in short term were effective for improving the consumption of added salt in adolescents.
\end{abstract}

Keywords: Sodium; Food and Nutrition Education; Adolescent. 